\title{
Disquinesia tardía inducida por neurolépticos y discapacidad en usuarios de larga estancia del Hospital Psiquiátrico Dr. Roberto Chacón Paut
}

José Rodolfo Gutiérrez -Villalobos.

\begin{abstract}
Justificación y objetivo: Los usuarios de larga estancia del Hospital Dr. Roberto Chacón Paut reciben tratamientos prolongados con neurolépticos, lo cual los expone a sufrir disquinesia tardía (DT). Este estudio tiene como objetivo determinar la prevalencia de DT inducida por neurolépticos, la discapacidad que genera, los factores de riesgo de sufrir disquinesia y sus efectos discapacitantes en esta población.
\end{abstract}

\begin{abstract}
Métodos: A 173 usuarios, se les administraron los instrumentos: Criterios de investigación DSM-IV para DT inducida por neurolépticos, Escala de movimientos involuntarios anormales e Indice de Barthel, con el fin de determinar la prevalencia de DT y la discapacidad por movimientos anormales y para ABVD. Se utilizó un análisis de regresión logística para establecer los factores de riesgo de sufrir DT y sus efectos discapacitantes.

Resultados: La prevalencia general de DT fue del 54\%. Este porcentaje fue superior en mujeres y aumentó en los grupos de mayor edad y con tratamientos prolongados. Las mujeres presentaron discapacidad más severa que los varones. La edad avanzada y años de tratamiento con neurolépticos, se asociaron con una discapacidad menos severa. Se encontró un riesgo significativo de sufrir DT y discapacidad moderada por movimientos anormales, 1,06 veces más frecuente por cada año de tratamiento, y de total dependencia para ABVD, 10,07 veces más frecuente en mujeres que en varones.
\end{abstract}

Conclusiones: Los años de tratamiento con neurolépticos fue el único factor de riesgo identificado en la ocurrencia de DT y discapacidad moderada por movimientos anormales. El sexo femenino se asoció con total dependencia para ABVD.

Descriptores: Disquinesia, prevalencia, riesgo, discapacidad, movimientos anormales, vida diaria.

Recibido: 08 de abril, 2003

Aceptado: 18 de junio, 2003

La DT es un trastorno asociado al uso de los neurolépticos, caracterizado por movimientos anormales e involuntarios en cara, boca, extremidades y tronco ${ }^{1}$. Se ha establecido que la edad avanzada, el sexo femenino y la duración del tratamiento con neurolépticos son los factores de mayor riesgo de sufrir DT ${ }^{1-9}$. Las formas severas generan una total discapacidad a los afectados, quienes con frecuencia requieren asistencia continua para satisfacer sus necesidades básicas ${ }^{10}$.

Aunque se ha propuesto la vitamina E para el tratamiento de la DT ${ }^{11,12}$, por su capacidad de neutralizar radicales libres asociados al trastorno, también se han sugerido las vitaminas B3,

Abreviaturas: ABVD, actividades básicas de la vida diaria. DSM-IV, manual diagnóstico y estadístico de los trastornos mentales. Asociación Psiquiátrica Americana. DT, disquinesia tardía.

Departamento de Psiquiatría. Hospital Dr. Roberto Chacón Paut.

Correspondencia: Apartado postal 171-2250, Tres Ríos.

E-mail:5gutiar@racsa.co.cr

102 AMC, Julio-Setiembre 2003, vol 45 (3)
B6 y C, y minerales como el manganeso ${ }^{13}$; otros informes ${ }^{14,15}$ indican que no existe un tratamiento único eficaz para la DT, y prevenir el trastorno sería la única forma de tratamiento ${ }^{16}$. Los neurolépticos atípicos se asocian con una menor prevalencia de DT ${ }^{17}$.

Los sujetos institucionalizados están más expuestos al uso de los neurolépticos y a sufrir DT ${ }^{18}$, la cual aparece entre el 50 y el $60 \%$ de los sujetos en estas condiciones ${ }^{6}$.

El propósito de esta investigación fue establecer la prevalencia de DT inducida por neurolépticos, la discapacidad que produce y los factores de riesgo de sufrir DT y sus efectos discapacitantes en los usuarios de larga estancia del Hospital Dr. Roberto Chacón Paut, quienes son individuos institucionalizados y medicados con neurolépticos por periodos prolongados ${ }^{19}$. 


\section{Materiales y métodos}

Se seleccionaron 173 sujetos de larga estancia, expuestos al uso crónico de neurolépticos. Se les administró el instrumento Criterios de investigación DSM-IV para disquinesia tardía inducida por neurolépticos. Este instrumento,compuesto por seis criterios de inclusión, identificó a los usuarios con DT y su prevalencia.

A los portadores de DT $(n=93)$ se les administró la Escala de movimientos involuntarios anormales y el Indice de Barthel. La escala evalúa la discapacidad por movimientos anormales, con una graduación de 0 (ausente), 1 (mínima), 2 (leve), 3 (moderada) y 4 (severa). Con el segundo instrumento, se identificó la discapacidad para ABVD; este mide niveles funcionales de autocuidado y movilidad y asigna un puntaje que permite distinguir el grado de severidad de la discapacidad. Un puntaje entre 81 y 100 indica independencia; 61 a 80 , poca dependencia; 41 a 60, marcada dependencia; 21 a 40, muy marcada dependencia; y 20 o menos, total dependencia. Los procedimientos más detallados se encuentran en el estudio de Gutiérrez ${ }^{20}$.

Se efectuó un análisis descriptivo porcentual para establecer la prevalencia de DT y sus efectos discapacitantes en relación con el sexo, la edad y la duración del tratamiento con neurolépticos. Con un análisis de regresión logística $(\mathrm{N}=173)$, se determinaron los factores de riesgo de sufrir DT, así como discapacidad por movimientos anormales y para ABVD. En los modelos de regresión, se utilizaron como variables independientes el sexo, la edad y la duración del tratamiento con neurolépticos, y se determinó su vínculo con las variables dependientes, prevalencia de DT, discapacidad leve, moderada y severa, poca dependencia, marcada dependencia, muy marcada dependencia y total dependencia. Se usó un nivel de significancia de $<0,05$.

Las autoridades médicas del hospital autorizaron el estudio y asumieron el consentimiento informado. Se contó con el aval del Comité Ético Científico de la Vicerrectoría de Docencia de la Universidad de Costa Rica.

El registro de datos se efectuó en el período comprendido entre el 4 y el 29 de junio de 2001.

\section{Resultados}

Los usuarios estuvieron medicados con uno o más de los siguientes neurolépticos: thioridazina, flufenazina, levomepromazina, clorpromazina y haloperidol; la vía de administración fue la oral, excepto en el caso de la flufenazina, que se administró por vía parenteral (intramuscular).

Se encontraron 93 usuarios portadores de DT, 66 varones y 27 mujeres, con una edad promedio de 52 años (rango de 2286) y un promedio de 23 años de tratamiento con neurolépticos (rango de 2-45). En 80 usuarios no se detectó DT. La prevalencia general de DT fue del $54 \%$, el $52 \%$ en los varones y el $60 \%$ en las mujeres.

\section{Cuadro 1.}

Distribución absoluta de usuarios y prevalencia relativa de DT según sexo, edad y duración del tratamiento con neurolépticos $(\mathrm{N}=173)$. Hospital Dr. R. Chacón Paut.

\begin{tabular}{|c|c|c|c|c|c|c|}
\hline & \multicolumn{3}{|c|}{ Hombres } & \multicolumn{3}{|c|}{ Mujeres } \\
\hline & $\mathrm{N}$ & $\mathrm{n}$ & $\%$ & $\mathrm{~N}$ & $\mathrm{n}$ & $\%$ \\
\hline & \multicolumn{6}{|c|}{ Edad } \\
\hline$<$ de 39 años $(\mathrm{N}=33)$ & 26 & 10 & 38 & 7 & 4 & 57 \\
\hline $40-49(\mathrm{~N}=52)$ & 39 & 18 & 46 & 13 & 6 & 46 \\
\hline $50-59(\mathrm{~N}=26)$ & 16 & 11 & 69 & 10 & 7 & 70 \\
\hline $60-69(\mathrm{~N}=46)$ & 36 & 18 & 50 & 10 & 6 & 60 \\
\hline $70 y>(N=16)$ & 11 & 9 & 82 & 5 & 4 & 80 \\
\hline \multicolumn{7}{|l|}{ Años de tratamiento } \\
\hline$<\operatorname{de} 19(\mathrm{~N}=53)$ & 42 & 13 & 31 & 11 & 4 & 36 \\
\hline $20-29(\mathrm{~N}=87)$ & 58 & 36 & 62 & 29 & 18 & 62 \\
\hline $30-39(\mathrm{~N}=24)$ & 19 & 10 & 53 & 5 & 5 & 100 \\
\hline $40 y>(N=9)$ & 9 & 7 & 78 & 0 & 0 & 0 \\
\hline
\end{tabular}

Ambos sexos registraron la mayor prevalencia de DT en los mayores de 70 años, $82 \%$ en los varones y un $80 \%$ en las mujeres. Según los años de tratamiento con neurolépticos, los varones presentaron una prevalencia de DT del 78\%, en los que tenían 40 o más años de tratamiento, en tanto en las mujeres fue del $100 \%$ en las que tenían entre 30 y 39 años de tratamiento (Cuadro 1).

Un $56 \%$ de los varones mostró discapacidad moderada por movimientos anormales, y el $48 \%$ de las mujeres, discapacidad severa. Un mayor porcentaje de usuarios presentó discapacidad moderada por movimientos anormales, según la edad y años de tratamiento con neurolépticos. Los mayores porcentajes de usuarios con esta discapacidad fueron un $78 \%$ en los que tenían de 50 a 59 años de edad y un $86 \%$ en los que tenían 40 años o más de tratamiento (Cuadro 2).

Un mayor porcentaje de mujeres $(59 \%)$ presentó total dependencia para $\mathrm{ABVD}$, mientras que los varones se distribuyeron en porcentajes similares a lo largo de todas las categorías. De acuerdo con la edad y años de tratamiento con neurolépticos, el mayor porcentaje de usuarios mostró poca dependencia para ABVD: un $44 \%$ entre los que tenían de 50 a 59 años de edad y un $57 \%$ en quienes tenían 40 años o más de tratamiento (Cuadro 3).

El cuadro 4 resume los resultados de la regresión logística e incluye solamente las variables que demostraron alguna significancia.

Los años de tratamiento con neurolépticos se asociaron con la ocurrencia de DT y discapacidad moderada por movimientos anormales; el riesgo de sufrir estos trastornos, se incrementa 1,06 veces con cada año de tratamiento. El sexo femenino se asoció con total dependencia para ABVD; con un riesgo 10,07 veces más frecuente en el sexo femenino. 


\begin{tabular}{|c|c|c|c|c|c|c|}
\hline \multicolumn{7}{|c|}{$\begin{array}{c}\text { Cuadro } 2 \text {. } \\
\text { Distribución absoluta y porcentual de } \\
\text { discapacidad por movimientos anormales según } \\
\text { severidad, sexo, edad y duración del tratamiento } \\
\text { con neurolépticos, en usuarios con DT ( } \mathrm{n}=93) \text {. } \\
\text { Hospital Dr. R. Chacón Paut. }\end{array}$} \\
\hline & \multicolumn{6}{|c|}{ Discapacidad } \\
\hline & \multicolumn{2}{|c|}{ Leve } & \multicolumn{2}{|c|}{ Moderada } & \multicolumn{2}{|c|}{ Severa } \\
\hline \multicolumn{7}{|l|}{ Sexo } \\
\hline Hombres $(n=66)$ & 9 & 14 & 37 & 56 & 20 & 30 \\
\hline Mujeres $(n=27)$ & 3 & 11 & 11 & 41 & 13 & 48 \\
\hline \multicolumn{7}{|l|}{ Edad } \\
\hline$<$ de 39 años $(n=14)$ & 1 & 7 & 7 & 50 & 6 & 43 \\
\hline $40-49(n=24)$ & 4 & 16 & 10 & 42 & 10 & 42 \\
\hline $50-59(n=18)$ & 2 & 11 & 14 & 78 & 2 & 11 \\
\hline $60-69(n=24)$ & 5 & 21 & 8 & 33 & 11 & 46 \\
\hline $70 y>(n=13)$ & 0 & 0 & 9 & 69 & 4 & 31 \\
\hline \multicolumn{7}{|l|}{ Años de tratamiento } \\
\hline$<$ de $19(\mathrm{n}=17)$ & 1 & 6 & 8 & 47 & 8 & 47 \\
\hline $20-29(n=54)$ & 6 & 11 & 27 & 50 & 21 & 39 \\
\hline $30-39(n=15)$ & 4 & 26 & 7 & 47 & 4 & 27 \\
\hline $40 y>(n=7)$ & 1 & 14 & 6 & 86 & 0 & 0 \\
\hline
\end{tabular}

Cuadro 3.

Distribución absoluta y porcentual de discapacidad para actividades básicas de la vida diaria, según grado de dependencia, sexo, edad y duración del tratamiento con neurolépticos en usuarios con DT ( $n=93)$. Hospital Dr. R. Chacón Paut.

\begin{tabular}{|c|c|c|c|c|c|c|c|c|}
\hline & \multicolumn{8}{|c|}{ Dependencia } \\
\hline & \multicolumn{2}{|c|}{ Poca } & \multicolumn{2}{|c|}{ Marcada } & \multicolumn{2}{|c|}{ Muy marcada } & \multicolumn{2}{|c|}{ Total } \\
\hline & $\mathrm{n}$ & $\%$ & $\mathrm{n}$ & $\%$ & $\mathrm{n}$ & $\%$ & $\mathrm{n}$ & $\%$ \\
\hline \multicolumn{9}{|l|}{ Sexo } \\
\hline Hombres $(n=66)$ & 22 & 33 & 18 & 27 & 19 & 29 & 7 & 11 \\
\hline Mujeres (n=27) & 7 & 26 & 1 & 4 & 3 & 11 & 16 & 59 \\
\hline \multicolumn{9}{|l|}{ Edad } \\
\hline$<$ de 39 años $(n=14)$ & 2 & 14 & 2 & 14 & 5 & 36 & 5 & 36 \\
\hline $40-49(n=24)$ & 5 & 21 & 3 & 13 & 8 & 33 & 8 & 33 \\
\hline $50-59(n=18)$ & 8 & 44 & 5 & 28 & 3 & 17 & 2 & 11 \\
\hline $60-69(n=24)$ & 10 & 42 & 8 & 33 & 1 & 4 & 5 & 21 \\
\hline $70 y>(n=13)$ & 4 & 31 & 1 & 8 & 5 & 38 & 3 & 23 \\
\hline \multicolumn{9}{|l|}{ Años de tratamiento } \\
\hline$<$ de $19(\mathrm{n}=17)$ & 4 & 23 & 4 & 23 & 4 & 23 & 5 & 29 \\
\hline $20-29(n=54)$ & 14 & 26 & 9 & 17 & 14 & 26 & 17 & 31 \\
\hline $30-39(n=15)$ & 7 & 47 & 5 & 33 & 2 & 13 & 1 & 7 \\
\hline $40 y>(n=7)$ & 4 & 57 & 1 & 14 & 2 & 29 & 0 & 0 \\
\hline
\end{tabular}

\section{Discusión}

La prevalencia general de DT (54\%) se sitúa dentro del rango usualmente aceptado del 50-60\% para individuos crónicamente institucionalizados ${ }^{6}$. En el Hospital Nacional Psiquiátrico de Costa Rica y en el Hospital Psiquiátrico de Berreshid, en Marruecos, se han reportado prevalencias de, 63.9 y del $63 \%$, respectivamente ${ }^{21,22}$, mientras que en hospitales de Hong Kong y Shanghai se han reportado de $9.3 \%$ y $8.4 \%$. 23.24 . Los autores asocian estas bajas prevalencias con el uso de neurolépticos en dosis bajas.

Las prevalencias de los varones (52\%) y de las mujeres $(60 \%)$ también se sitúan dentro del rango referido en la bibliografía ${ }^{6}$; sin embargo, la de las mujeres se ubicó en el límite superior del rango y a ocho puntos porcentuales sobre la de los varones. Se ha referido que las mujeres están más expuestas a sufrir $\mathrm{DT}^{25}$, debido a una disminución de los estrógenos por el bloqueo dopaminérgico ${ }^{19,26}$. Los estrógenos modulan el comportamiento mediado por la dopamina y protegen contra el estrés oxidativo de la neurona expuesta prolongadamente a los neurolépticos ${ }^{26}$. Otros registros indican que los varones y las mujeres están igualmente propensos a sufrir DT ${ }^{18}$.

A pesar de que ambos sexos presentaron, según la edad, prevalencias variables en los diferentes rangos, las más altas se registraron en los mayores de 70 años; en general, a mayor edad, tanto hombres como mujeres están expuestos a sufrir DT en mayor proporción. Se informa que los ancianos son muy vulnerables a sufrir DT y que la frecuencia del trastorno aumenta con la edad ${ }^{1,4}$; otros reportes señalan que el mayor riesgo de sufrir el trastorno se presenta en mujeres ancianas y en hombres jóvenes ${ }^{7}$. En esta investigación se hallaron prevalencias superiores al $60 \%$ y $80 \%$, en los mayores de 50 y 70 años, respectivamente.

Por años de tratamiento con neurolépticos, se encontraron prevalencias crecientes para ambos sexos, conforme aumentó el número de años de tratamiento; tal incremento concuerde con la bibliografía $1,3,5,5,27,28$. Se citan prevalencias de DT del $16 \%$ en pacientes con menos de tres meses de exposición a los neurolépticos; un $29 \%$, entre tres y doce meses; un 30\%, entre uno y doce años; y un $41 \%$ en más de 10 años ${ }^{1}$. En esta investigación las prevalencias fueron superiores al $41 \%$, a partir de los 20 años de tratamiento.

En cuanto a la discapacidad por movimientos anormales y para $\mathrm{ABVD}$, las mujeres presentaron una más severa que los varones, lo que indica que estarían más expuestas a sufrir los efectos discapacitantes de la DT; esto podría estar determinado por la misma predisposición biológica a sufrir DT ${ }^{9}$. La edad y años de tratamiento registran una notoria relación con una discapacidad moderada por movimientos anormales, lo que indicaría que tales variables se asocian con un 


\begin{tabular}{|c|c|c|c|}
\hline \multicolumn{4}{|c|}{$\begin{array}{c}\text { Cuadro } 4 . \\
\text { Riesgo de sufrir DT y sus efectos discapacitantes } \\
\text { en usuarios de larga estancia }(\mathrm{N}=173) \text {. } \\
\text { Hospital Dr. R. Chacón Paut. }\end{array}$} \\
\hline $\begin{array}{l}\text { Variable } \\
\text { dependiente }\end{array}$ & $\begin{array}{c}\text { Variable } \\
\text { independiente }\end{array}$ & Significancia & Exp. (B) \\
\hline Disquinesia tardía & $\begin{array}{c}\text { Sexo } \\
\text { Edad } \\
\text { Años de tratamiento }\end{array}$ & $\begin{array}{l}0,4894 \\
0,4022 \\
0,0008\end{array}$ & $\begin{array}{l}1,2887 \\
1,0106 \\
1,0698\end{array}$ \\
\hline $\begin{array}{l}\text { Discapacidad } \\
\text { moderada }\end{array}$ & $\begin{array}{c}\text { Sexo } \\
\text { Edad } \\
\text { Años de tratamiento }\end{array}$ & $\begin{array}{l}0,4697 \\
0,6163 \\
0,0075\end{array}$ & $\begin{array}{l}0,7435 \\
1,0073 \\
1,0626\end{array}$ \\
\hline Total dependencia & $\begin{array}{c}\text { Sexo } \\
\text { Edad } \\
\text { Años de tratamiento }\end{array}$ & $\begin{array}{c}0,00001 \\
0,4301 \\
0,9348\end{array}$ & $\begin{array}{r}10,0795 \\
0,9844 \\
0,9974\end{array}$ \\
\hline
\end{tabular}

incremento de esta discapacidad; en relación con discapacidad para ABVD, las dos variables se asociaron en un mayor porcentaje a poca dependencia.

El análisis de regresión corroboró que los años de tratamiento con neurolépticos constituyeron un factor de riesgo en la ocurrencia de DT y discapacidad moderada por movimientos anormales; en tanto el sexo femenino fue el factor de riesgo en la ocurrencia de total dependencia para ABVD.

Se puede concluir que los usuarios de larga estancia del Hospital Dr. Roberto Chacón Paut presentan una prevalencia de DT que se ubica en los rangos referidos en la bibliografía, y que los años de tratamiento con neurolépticos constituyen el único factor de riesgo de sufrir el trastorno. La discapacidad que genera la DT es más severa en mujeres que en varones, y el sexo femenino y los años de tratamiento con neurolépticos son factores significativos en su ocurrencia; por lo tanto, se deben controlar los períodos de tratamiento de estos usuarios, justificar racionalmente el uso a largo plazo de los neurolépticos, y prestar especial cuidado y atención a las mujeres que reciben estos fármacos. Los neurolépticos atípicos son una opción de tratamiento que debe considerarse ${ }^{8,17,29,30}$.

\section{Abstract}

Background and aim: To establish the prevalence of tardive dyskinesia (TD) induced by neuroleptics. The risk factors involved and the disability effects in chronic users at the Chacón Paut Psychiatric Hospital.

Method: 173 patients were studied. The DSM-IV Investigation Criteria for Tardive Dyskinesia induced by neuroleptics, Abnormal involuntary movement scale and Barthel Index were administrated to determine prevalence of $\mathrm{TD}$, disability for abnormal movements and for basic activities of daily life. Regression logistic analysis was used to determine the risk factors of suffering TD and disability for abnormal movements and for basic activities of daily life
Results: The general prevalence of TD was 54\%. Women, old age groups and people with long time treatment presented a higher prevalence than general. Women registered disability for abnormal movements and for basic activities of daily life more severely than men. Age and years of treatment with neuroleptics were associated with less severe disability. The risk of suffering TD and moderate disability for abnormal movements was 1.06 , which was more frequent for every year of treatment. The risk of suffering total dependence for basic activities of daily life was 10.07 once again, more frequently in women than in men.

Conclusions: Treatment duration with neuroleptics were a risk factor of suffering TD and moderate disability for abnormal movements. Female sex was a risk factor in occurrence to total dependence for the basic activities of daily life.

\section{Referencias}

1. Bruce, A. Tardive Dyskinesia. Virtual Hospital: Clinical Psychopharmacology Seminar. http://www.vh.org/Providers/Conferences/ CPS/08.html 1996; 1-15.

2. Smith, J. Baldessarini,R. Changes in prevalence, severity, and recovery in tardive dyskinesia with age. Arch Gen Psychiatry 1980; 37: 13681373.

3. Chouinard, D. A 5 years prospective longitudinal study of tardive dyskinesia. Factors predicting appearance of new cases. J of Clin Psychopharmacology 1988; 8(4):21-25.

4. Yassa, R. Nastase, Ch. Dupond, D. Thibeau, M. Tardive dyskinesia in elderly psychiatric patients:A five year Study. American J. Psychiatry 1992; 149:1206-2011.

5. Stoessl, J. Drogas que causan distonía. Distonía Dialogue, 1996; 19(1):1-3.

6. Kaplan, H. Sinopsis de Psiquiatría. Octava edición. Editorial Masson. Madrid, España, 1999; 1094-1095.

7. Van Os, J. Wals,E. Van Horn,E. Tattan, T. Bale, R. Thompson,SG. Tardive dyskinesia in psychosis:Are woman really more at risk? Acta Psychiatr Scand 1999; 99(4): 288-293.

8. Kane, J. Reducing the risk of tardive dyskinesia. Zeneca Pharmaceuticals 1997; 1(2): 13-16.

9. Kane, J. Smith, J. Tardive dyskinesia. Arch Gen Psychiatry 1982; 39: 473-481.

10. Gardos, G. Cole, J. Salomon,M. Schniebolk S. Clinical forms of severe tardive dyskinesia. Am J Psychiatry 1987; 144(7): 895-902.

11. Elkashef, A. Ruskin, P. Bacher, N. Barrett, D. Vitamin E in the treatment of tardive dyskinesia. Am J Psychiatry 1990; 147(4): 505-506.

12. Pascual, A. Pérez, V. Lozano, I. Tratamiento de las discinesias tardías con vitamina E. Psiquis 1998; 19(8): 337-342.

13. Edelson, S. Tardive Dyskinesia. Center for the Study of Autism. http//www.autism.org/tardiv.html 1995; 1.

14. Jeste, D. Wyatt, R. Therapeutic strategies against tardive dyskinesia. Arch Gen Psychiatry 1992; 39:803-816.

15. Sachdev, PS. The current status of tardive dyskinesia. Aust NZ J Psychiatry 2000; 34(3):355-369.

16. Arce, G. Antipsicóticos. Departamento de Salud Mental. Caja Costarricense de Seguro Social,San José,Costa Rica, 1997; 14-15.

17. Uriarte, V. Psicofarmacología. Editorial Trillas. México,1992; 82-138.

18. Asociación Psiquiátrica Americana. DSM-IV. Manual diagnóstico y estadístico de los trastornos mentales. Editorial Masson S.A. Barcelona, España, 1995; 752-765. 
19. Gutiérrez, J.R. Perfil clínico y farmacológico de los pacientes del hospital Chacón Paut. Manuscrito no publicado. San José, Costa Rica, 1989; 1-27.

20. Gutiérrez,JR. Disquinesia tardía inducida por neurolépticos y discapacidad en usuarios de larga estancia del Hospital Psiquiátrico Dr. Roberto Chacón Paut. Tesis sin publicar, sometida a la consideración de la comisión del programa de estudios de postgrado en estudios interdisciplinarios sobre discapacidad para optar al grado de magíster scientiae. Universidad de Costa Rica. San José, Costa Rica,2002.

21. Jiménez, F. Perfil psicofarmacológico comparativo del esquizofrénico crónico en el Hospital Nacional Psiquiátrico. Revista Fármacos 1988; 4(1): 41-50

22. Moussaoui, D. Douki, S. Bentousni, G. Otarid, A. Chorfi, M. Mamou, A. Epidemiology of the tardive dyskinesia in the Maghreb. Encephale $1988 ; 14: 203-208$.

23. Chiu, H. Shum, P. Lau, J. Lam, L. Lee, S. Prevalence of tardive dyskinesia, tardive dystonia and respiratory dyskinesia among chinese psychiatric patients in Hong Kong. Am J Psychiatry 1992; 149(8): 10811085 .
24. Ko, G. Shang, L. Yan, W. Zhang, M. Buchner, D. Xia, Zhen. Wyatt, R. Jeste, D. The Shanghai 800:Prevalence of tardive dyskinesia in a chinese psychiatric hospital. Am J Psychiatry 1998; 146(3): 387-389.

25. Barnes,RE. Braude, W. Akathisia variants and tardive dyskinesia. Arch Gen Psychiatry 1985; 42:874-878.

26. Turrone P, Seeman MV, Silvestri S. Estrogen activation and tardive dyskinesia. Can J Psychiatry 2000; 45 (3):288-90.

27. National Alliance for The Mentally (NAMI). Tardive dyskinesia. http://www.nami org/helpline /tardys.htm 1996; 1-3.

28. Mc Greadie, RG. Ohaeri, JU. Movement disorder in never and minimally treated Nigerian schizophrenic patients. Br J of Psychiatry 1994; 164: 184-189.

29. Tollefson, G. Beasley, Ch. Tamura, R. Tran, P. Potvin, J. Blind controlled long-term study of the comparative incidence of treatment-emergent tardive dyskinesia with olanzapina or haloperidol. Am J Psychiatry 1997; 154(9): 1248-1254.

30. Glazer, W. Expected incidence of tardive dyskinesia associated with atypical antipsychotics J Clin Psychiatry 2000; 61 (suppl 4): 21-26. 